\title{
Microbicide trial screeches to a halt
}

A phase III clinical trial to test the effectiveness of the HIV microbicide Ushercell was recently stopped because interim analysis indicated that the rate of infection with HIV in women using the microbicide was higher than the rate of infection in those using the placebo.

Ushercell, which was being developed by the nonprofit reproductive health organization CONRAD, is a cellulose sulfate compound that blocks viral entry into the cell. Its inhibitory function is thought to be due to the fact that it is polyanionic and can interfere with the charged interaction between positive charges on the viral envelope and negative charges on the cell surface.

The women who enrolled in the doubleblinded randomized trial, which was being conducted in India, Uganda, Benin, and South Africa, were randomly assigned to use either Ushercell, which comes in gel form and is inserted vaginally up to one hour before intercourse, or a placebo gel. The trial was halted when the independent group of experts analyzing the interim data noted a higher rate of HIV infection in women using Ushercell than in women using the placebo.

The interim results of the trial came as a shock to researchers in the field, including those conducting the study, such as Lut Van Damme, who led the trial and told the JCI, "We are extremely disappointed by the results of the trial and were very surprised by the increased risk of becoming infected with HIV."
Much of the surprise about the trial results arose because no safety concerns had been noted in the 11 previously completed safety and contraceptive trials of Ushercell involving more than 500 patients in Africa, India, Belgium, and the United States.

This lack of prior indication that Ushercell use might raise safety issues contrasts with the case of the detergent nonoxynol-9, another microbicide that failed in clinical trials because it increased the rate of infection with HIV. Going into the clinical trials, nonoxynol-9 was known to create lesions in the mucosal lining of the vagina; ultimately these lesions were shown to result in the increased rate of HIV infection. By contrast, no data regarding Ushercell even hinted that its use might present problems.

Although researchers involved in the trial currently have no idea why Ushercell increased the rate of infection with HIV, CONRAD is determined to establish why this happened. One of the ways in which they are hoping to address this issue is to "put together a blinded panel of compounds, including cellulose sulfate, and send it out to various individuals working on different models and see if they can come up with any clues," according to Henry Gabelnick, CONRAD's executive director (1). Van Damme added that they "hope to learn from the experience and perhaps understand how preclinical models differ from the clinical situation." In

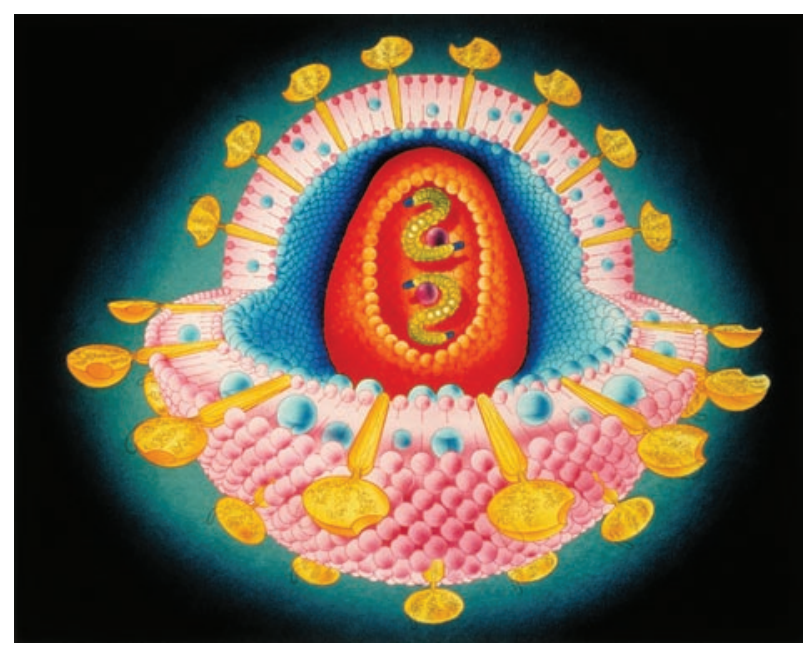

\section{Figure 1}

In Sub-Saharan Africa, women are more at risk than men of becoming infected with HIV, and $57 \%$ of people living with HIV are women. Image credit: Photo Researchers Inc.

the view of Michael Lederman, an expert in HIV microbicide research at Case Western Reserve University, the fact that "they are clearly committed to learning as much as possible about why cellulose sulfate failed or was harmful will only help the field."

Other researchers are concerned about the impact that the failure of this trial will have on the development of other microbicides, the most advanced of which are Carraguard (Population Council) and PRO 2000 (Indevus Pharmaceuticals), both polyanionic microbicides in phase III clinical trials. The trial with Carraguard was completed at the end of March 2007, and although the results will not be available until the end of 2007 at the earliest, the independent group of experts monitoring the interim results of the trial has not noted any safety issues. Despite the assumption that Carraguard and PRO 2000 will be safe, some researchers are asking whether the microbicides currently being tested in clinical trials are the best ones; many are favoring a move to a more specific approach, such as the topical application of antiviral drugs (2). Indeed, as Lederman told the JCI, "The good news is that there are microbicide candidates in the pipeline that target critical viral or host elements and there is reason to be more optimistic about their chances for success."

Another criticism of the current approach to developing microbicides is that the process is not streamlined enough: experts have questioned whether 3 polyanionic microbicides needed to be tested in phase III clinical trials or whether the best candidate should have been singled out at an earlier stage of development (1). However, others, such as Gabelnick, counter that, "We don't know enough to say these [drugs that target HIV in the same way] are all the same" (1).

The one thing that researchers do agree on is the need for an effective microbicide, as women in Sub-Saharan Africa and elsewhere are more likely than men to become infected with HIV, and many social issues in these regions do not allow them to easily protect themselves from infection.

\section{Karen Honey}

1. Check, E. 2007. Scientists rethink approach to HIV gels. Nature. 446:12.

2. Gawrylewski, A. 2007. Failure of HIV microbicide raises concerns. The scientist. http://www.the-scientist.com/news/home/52861/. 